\title{
Universiteit
}

Leiden

The Netherlands

\section{Effective mass and tricritical point for lattice fermions localized by a} random mass

Medvedyeva, M.V.; Tworzydlo, J.; Beenakker, C.W.J.

\section{Citation}

Medvedyeva, M. V., Tworzydlo, J., \& Beenakker, C. W. J. (2010). Effective mass and tricritical point for lattice fermions localized by a random mass. Physical Review B, 81(21), 214203. doi:10.1103/PhysRevB.81.214203

Version: $\quad$ Not Applicable (or Unknown)

License: $\quad$ Leiden University Non-exclusive license

Downloaded from: https://hdl.handle.net/1887/59990

Note: To cite this publication please use the final published version (if applicable). 


\title{
Effective mass and tricritical point for lattice fermions localized by a random mass
}

\author{
M. V. Medvedyeva, ${ }^{1}$ J. Tworzydło, ${ }^{2}$ and C. W. J. Beenakker ${ }^{1}$ \\ ${ }^{1}$ Instituut-Lorentz, Universiteit Leiden, P.O. Box 9506, 2300 RA Leiden, The Netherlands \\ ${ }^{2}$ Institute of Theoretical Physics, University of Warsaw, Hoża 69, 00-681 Warsaw, Poland \\ (Received 10 April 2010; revised manuscript received 28 May 2010; published 21 June 2010)
}

\begin{abstract}
This is a numerical study of quasiparticle localization in symmetry class $B D$ (realized, for example, in chiral $p$-wave superconductors), by means of a staggered-fermion lattice model for two-dimensional Dirac fermions with a random mass. For sufficiently weak disorder, the system size dependence of the average (thermal) conductivity $\sigma$ is well described by an effective mass $M_{\text {eff }}$, dependent on the first two moments of the random mass $M(r)$. The effective mass vanishes linearly when the average mass $\bar{M} \rightarrow 0$, reproducing the known insulator-insulator phase boundary with a scale invariant dimensionless conductivity $\sigma_{c}=1 / \pi$ and critical exponent $\nu=1$. For strong disorder a transition to a metallic phase appears, with larger $\sigma_{c}$ but the same $\nu$. The intersection of the metal-insulator and insulator-insulator phase boundaries is identified as a repulsive tricritical point.
\end{abstract}

DOI: 10.1103/PhysRevB.81.214203

PACS number(s): 72.15.Rn, 73.20.Jc, 74.25.fc, 74.78.Na

\section{INTRODUCTION}

Superconductors with neither time-reversal symmetry nor spin-rotation symmetry (for example, having chiral $p$-wave pairing) still retain one fundamental symmetry: the chargeconjugation (or particle-hole) symmetry of the quasiparticle excitations. Because of this symmetry, quasiparticle localization in a disordered chiral $p$-wave superconductor is in a different universality class than in a normal metal. ${ }^{1}$ The difference is particularly interesting in two dimensions, when the quantum Hall effect governs the transport properties. The electrical quantum Hall effect in a normal metal has the thermal quantum Hall effect as a superconducting analog, ${ }^{2-4}$ with different scaling properties because of the particle-hole symmetry.

The thermal quantum Hall transition is analogous to the electrical quantum Hall transition at the center of a Landau level but the scaling of the thermal conductivity $\sigma$ near the phase boundary is different from the scaling of the electrical conductivity because of the particle-hole symmetry. A further difference between these two problems appear if the superconducting order parameter contains vortices. ${ }^{2,5,6} \mathrm{~A}$ vortex contains a Majorana bound state at zero excitation energy in the weak-pairing regime. ${ }^{7,8}$ A sufficiently large density of Majorana bound states allows for extended states at the Fermi level, with a thermal conductivity increasing $\propto \ln L$ with increasing system size $L .^{3}$ This so-called thermal metal has no counterpart in the electronic quantum Hall effect.

The Bogoliubov-De Gennes Hamiltonian of a disordered chiral $p$-wave superconductor can be approximated at low energies by a Dirac Hamiltonian with a random mass (see Sec. II). For that reason, it is convenient to parameterize the phase diagram in terms of the average mass $\bar{M}$ and the fluctuation strength $\delta M$. As indicated in Fig. 1, there are two types of phase transitions, ${ }^{10,11}$ a metal-insulator (M-I) transition upon decreasing $\delta M$ at constant $\bar{M}$ and an insulatorinsulator (I-I) transition upon decreasing $\bar{M}$ through zero at constant (not too large) $\delta M$. The I-I transition separates phases with a different value of the thermal Hall conductance while the M-I transition separates the thermal metal from the thermal insulator. Only the I-I transition remains if there are no vortices, or more generally, if there are no Majorana bound states. ${ }^{2,5,6}$ In the nomenclature of Ref. 5, the symmetry class is called $B D$ with Majorana bound states and $D$ without.

The primary purpose of our paper is to investigate, by numerical simulation, to what extent the scale dependence of localization by a random mass can be described in terms of an effective nonfluctuating mass: $\sigma(L, \bar{M}, \delta M)$ $=\sigma\left(L, M_{\mathrm{eff}}, 0\right)$, for some function $M_{\mathrm{eff}}(\bar{M}, \delta M)$. Because there is no other length scale in the problem at zero energy, $\sigma\left(L, M_{\text {eff }}, 0\right)$ can only depend on $L$ and $M_{\text {eff }}$ through the dimensionless combination $L M_{\text {eff }} U / \hbar \equiv L / \xi$. The effectivemass hypothesis thus implies one-parameter scaling: $\sigma(L, \bar{M}, \delta M)=\sigma_{0}(L / \xi)$. Two further implications concern the critical conductivity $\sigma_{c}$ (which is the scale invariant value of $\sigma$ on the phase boundary $\bar{M}=0$ ) and the critical exponent $\nu$ (governing the divergence of the localization length $\xi \propto \bar{M}^{-\nu}$ ).

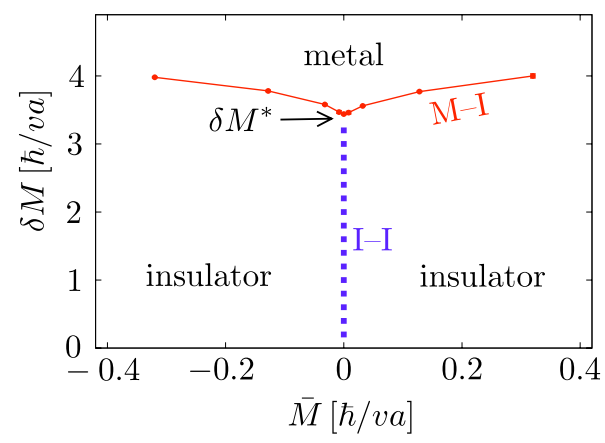

FIG. 1. (Color online) Phase diagram in symmetry class $B D$, calculated numerically from the lattice model of staggered fermions described in Sec. III. [A qualitatively similar phase diagram was calculated for a different model (Ref. 9) in Refs. 10 and 11]. The thermal conductivity decays exponentially $\propto e^{-L / \xi}$ in the localized phase and increases $\propto \ln L$ in the metallic phase. The thermal conductivity is scale invariant on the M-I phase boundary (solid line), as well as on the I-I phase boundary (dashed line). The M-I and I-I phase boundaries meet at the tricritical point $\delta M^{*}$. 
Both $\sigma_{c}$ and $\nu$ follow directly from the effective-mass hypothesis. By construction, the scaling function $\sigma_{0}$ is the conductivity of ballistic massless Dirac fermions, which has been calculated in the context of graphene. For a system with dimensions $L \times W$, and periodic boundary conditions in the transverse direction, it is given by ${ }^{12,13}$

$$
\begin{aligned}
& \sigma_{0}(L / \xi)=G_{0} \frac{L}{W} \sum_{n=-\infty}^{\infty} \cosh ^{-2} \sqrt{(2 \pi n L / W)^{2}+(L / \xi)^{2}} \\
& \stackrel{W \gg L}{\rightarrow} G_{0} \frac{1}{\pi} \int_{0}^{\infty} d q \cosh ^{-2} \sqrt{q^{2}+(L / \xi)^{2}} .
\end{aligned}
$$

A scale invariant conductivity

$$
\lim _{\xi \rightarrow \infty} \sigma_{0}(L / \xi) \equiv \sigma_{c}=G_{0} \frac{L}{W} \sum_{n=-\infty}^{\infty} \cosh ^{-2}(2 \pi n L / W)
$$

is reached for vanishing effective mass. In the limit of a large aspect ratio $W / L \gg 1$ we recover the known value $\sigma_{c}$ $=G_{0} / \pi$ of the critical conductivity for a random mass with zero average. ${ }^{14}$ The critical exponent $\nu=1$ follows by comparing the expansion of the conductivity

$$
\sigma(L, \bar{M}, \delta M)=\sigma_{c}+\left[L^{1 / \nu} \bar{M} f(\delta M)\right]^{2}+\mathcal{O}(\bar{M})^{4}
$$

in (even) powers of $\bar{M}$ with the expansion of the scaling function [Eq. (1.1)] in powers of $L$. This value for $\nu$ is aspect-ratio independent and agrees with the known result for the I-I transition. ${ }^{1}$

The description in terms of an effective mass breaks down for strong disorder. We find that the scaling function at the M-I transition differs appreciably from $\sigma_{0}$, with an aspectratio independent critical conductivity $\sigma_{c} \approx 0.4 G_{0}$. The critical exponent remains close to or equal to $\nu=1$ (in disagreement with earlier numerical simulations ${ }^{11}$ ).

The secondary purpose of our paper is to establish the nature of the tricritical point $\delta M^{*}$ at which the two insulating phases and the metallic phase meet. The existence of such a fixed point of the scaling flow is expected on the basis of general arguments ${ }^{5}$ but whether it is a repulsive or attractive fixed point has been a matter of debate. From the scale dependence of $\sigma$ near this tricritical point, we conclude that it is a repulsive fixed point (in the sense that $\sigma$ scales with increasing $L$ to larger values for $\delta M>\delta M^{*}$ and to smaller values for $\delta M<\delta M^{*}$ ). An attractive tricritical point had been suggested as a possible scenario, ${ }^{15,16}$ in combination with a repulsive critical point at some $\delta M^{* *}<\delta M^{*}$. Our numerics does not support this scenario.

The outline of this paper is as follows. In the next two sections we introduce the Dirac Hamiltonian for chiral $p$-wave superconductors and the lattice fermion model that we use to simulate quasiparticle localization in symmetry class $B D$. We only give a brief description, referring to the Appendix and Ref. 17 for a more detailed presentation of the model. The scaling of the thermal conductivity and the localization length near the insulator-insulator and metal-insulator transitions are considered separately in Secs. IV and V, re- spectively. The tricritical point, at which the two phase boundaries meet, is studied in Sec. VI. We conclude in Sec. VII.

\section{CHIRAL $p$-WAVE SUPERCONDUCTORS}

The quasiparticles in a superconductor have electron and hole components $\psi_{e}, \psi_{h}$ that are eigenstates, at excitation energy $\varepsilon$, of the Bogoliubov-De Gennes equation

$$
\left(\begin{array}{cc}
H_{0}-E_{F} & \Delta \\
\Delta^{\dagger} & -H_{0}^{*}+E_{F}
\end{array}\right)\left(\begin{array}{l}
\psi_{e} \\
\psi_{h}
\end{array}\right)=\varepsilon\left(\begin{array}{l}
\psi_{e} \\
\psi_{h}
\end{array}\right)
$$

In a chiral $p$-wave superconductor the order parameter $\Delta$ $=\frac{1}{2}\left\{\chi(\boldsymbol{r}), p_{x}-i p_{y}\right\}$ depends linearly on the momentum $\boldsymbol{p}=$ $-i \hbar \partial / \partial \boldsymbol{r}$, so the quadratic terms in the single-particle Hamiltonian $H_{0}=p^{2} / 2 m+U(\boldsymbol{r})$ may be neglected near $p=0$.

For a uniform order parameter $\chi(\boldsymbol{r})=\chi_{0}$, the quasiparticles are eigenstates of the Dirac Hamiltonian

$$
H_{\text {Dirac }}=v\left(p_{x} \sigma_{x}+p_{y} \sigma_{y}\right)+v^{2} M(\boldsymbol{r}) \sigma_{z}
$$

with velocity $v=\chi_{0}$ and mass $M=\left(U-E_{F}\right) / \chi_{0}^{2}$ (distinct from the electron mass $m$ ). The Pauli matrices are

$$
\sigma_{x}=\left(\begin{array}{ll}
0 & 1 \\
1 & 0
\end{array}\right), \quad \sigma_{y}=\left(\begin{array}{cc}
0 & -i \\
i & 0
\end{array}\right), \quad \sigma_{z}=\left(\begin{array}{cc}
1 & 0 \\
0 & -1
\end{array}\right) .
$$

The particle-hole symmetry for the Dirac Hamiltonian is expressed by

$$
\sigma_{x} H_{\text {Dirac }}^{*} \sigma_{x}=-H_{\text {Dirac }} .
$$

Randomness in the electrostatic potential $U(\boldsymbol{r})$ translates into randomness in the mass $M(\boldsymbol{r})=\bar{M}+\delta M(\boldsymbol{r})$ of the Dirac fermions. The sign of the average mass $\bar{M}$ determines the thermal Hall conductance, ${ }^{2-4}$ which is zero for $\bar{M}>0$ (strong pairing regime) and quantized at $G_{0}=\pi^{2} k_{B}^{2} T / 6 h$ for $\bar{M}<0$ (weak-pairing regime).

The Dirac Hamiltonian [Eq. (2.2)] provides a generic lowenergy description of the various realizations of chiral $p$-wave superconductors proposed in the literature: strontium ruthenate, ${ }^{18}$ superfluids of fermionic cold atoms, ${ }^{19,20}$ and ferromagnet-semiconductor-superconductor

heterostructures. ${ }^{21-23}$ What these diverse systems have in common is that they have superconducting order with neither time-reversal nor spin-rotation symmetry. Each of these systems is expected to exhibit the thermal quantum Hall effect, described by the phase diagram studied in this work.

\section{STAGGERED FERMION MODEL}

Earlier numerical investigations ${ }^{10,11,15,16}$ of the class $B D$ phase diagram were based on the Cho-Fisher network model. ${ }^{9}$ Here we use a staggered-fermion model in the same symmetry class, originally developed in the context of lattice gauge theory ${ }^{24,25}$ and recently adapted to the study of transport properties in graphene. ${ }^{17}$ An attractive feature of the lattice model is that, by construction, it reduces to the Dirac 
Hamiltonian on length scales large compared to the lattice constant $a$.

The model is defined on a square lattice in a strip geometry, extending in the longitudinal direction from $x=0$ to $x$ $=L=N_{x} a$ and in the transverse direction from $y=0$ to $y=W$ $=N_{y} a$. We use periodic boundary conditions in the transverse direction. The transfer matrix $\mathcal{T}$ from $x=0$ to $x=L$ is derived in Ref. 17, and we refer to that paper and to the Appendix for explicit formulas.

The dispersion relation of the staggered fermions,

$$
\tan ^{2}\left(k_{x} a / 2\right)+\tan ^{2}\left(k_{y} a / 2\right)+\left(\frac{M a v}{2 \hbar}\right)^{2}=\left(\frac{\varepsilon a}{2 \hbar v}\right)^{2}
$$

has a Dirac cone at wave vectors $|\boldsymbol{k}| a \ll 1$ which is gapped by a nonzero mass. Staggered fermions differ from Dirac fermions by the pole at the edge of Brillouin zone $\left(\left|k_{x}\right| \rightarrow \pi / a\right.$ or $\left.\left|k_{y}\right| \rightarrow \pi / a\right)$, which is insensitive to the presence of a mass. We do not expect these large-wave-number modes to affect the large-length scaling of the conductivity because they preserve the electron-hole symmetry.

The energy is fixed at $\varepsilon=0$ (corresponding to the Fermi level for the superconducting quasiparticles). The transfer matrix $\mathcal{T}$ is calculated recursively using a stable QR decomposition algorithm. ${ }^{26}$ An alternative stabilization method ${ }^{17}$ is used to recursively calculate the transmission matrix $t$. Both algorithms give consistent results but the calculation of $\mathcal{T}$ is more accurate than that of $t$ because it preserves the electronhole symmetry irrespective of round-off errors.

The random mass is introduced by randomly choosing values of $M$ on each site uniformly in the interval $(\bar{M}$ $-\delta M, \bar{M}+\delta M)$. Variations in $M(\boldsymbol{r})$ on the scale of the lattice constant introduce Majorana bound states, which place the model in the $B D$ symmetry class. ${ }^{27}$ In principle, it is possible to study also the class $D$ phase diagram (without Majorana bound states), by choosing a random mass landscape that is smooth on the scale of $a$. Such a study was recently performed, ${ }^{28}$ using a different model, ${ }^{29}$ to demonstrate the absence of the M-I transition in class $D .^{2,5,6}$ Since here we wish to study both the I-I and M-I transitions, we do not take a smooth mass landscape.

\section{SCALING NEAR THE INSULATOR-INSULATOR TRANSITION}

\section{A. Scaling of the conductivity}

In Fig. 2 we show the average (thermal) conductivity $\sigma$ $=(L / W)\left\langle\operatorname{Tr} t t^{\dagger}\right\rangle$ (averaged over some $10^{3}$ disorder realizations) as a function of $L$ for a fixed $\delta M$ in the localized phase. Data sets with different $\bar{M}$ collapse on a single curve upon rescaling with $\xi$. (In the logarithmic plot this rescaling amounts simply to a horizontal displacement of the entire data set.) The scaling curve (solid line in Fig. 2) is the effective-mass conductivity [Eq. (1.1)], with $M_{\text {eff }}=\hbar / v \xi$. Figure 3 shows the linear scaling of $\sigma$ with $(\bar{M} L)^{2}$ for small $\bar{M}$, as expected from Eq. (1.3) with $\nu=1$.

We have studied the aspect-ratio dependence of the critical conductivity $\sigma_{c}$. As illustrated in Fig. 4, the convergence

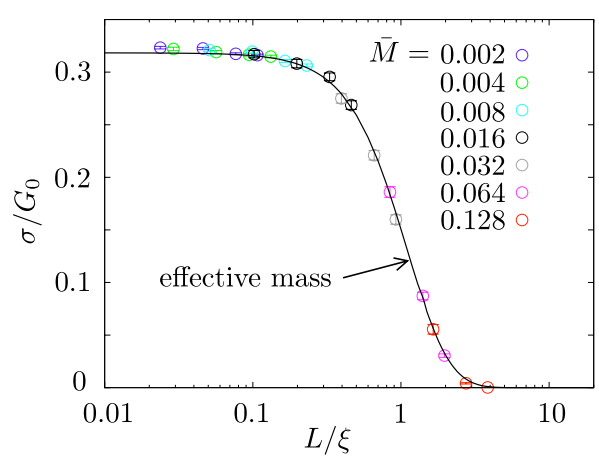

FIG. 2. (Color online) Average conductivity $\sigma$ (with error bars indicating the statistical uncertainty) at fixed disorder strength $\delta M$ $=2.5 \hbar / v a$, as a function of system size $L$. The aspect ratio of the disordered strip is fixed at $W / L=5$. Data sets at different values of $\bar{M}$ (listed in the figure in units of $\hbar / v a$ ) collapse upon rescaling by $\xi$ onto a single curve (solid line), given by Eq. (1.1) in terms of an effective mass $M_{\mathrm{eff}}=\hbar / v \xi$.

for $W / L \rightarrow \infty$ is to the value $\sigma_{c}=1 / \pi$ expected from Eq. (1.1). The conductivity of ballistic massless Dirac fermions also has an aspect-ratio dependence, ${ }^{13}$ given by Eq. (1.2) (for periodic boundary conditions). The comparison in Fig. 4 of $\sigma_{c}$ with Eq. (1.2) shows that $\sigma_{c}$ at the I-I transition follows quite closely this aspect-ratio dependence (unlike at the M-I transition discussed in Sec. V A).

\section{B. Scaling of the Lyapunov exponent}

The transfer matrix $\mathcal{T}$ provides an independent probe of the critical scaling through the Lyapunov exponents. The transfer-matrix product $\mathcal{T} \mathcal{T}^{\dagger}$ has eigenvalues $e^{ \pm \mu_{n}}$ with 0 $\leq \mu_{1} \leq \mu_{2} \leq \cdots$. The $n$th Lyapunov exponent $\alpha_{n}$ is defined by

$$
\alpha_{n}=\lim _{L \rightarrow \infty} \frac{\mu_{n}}{L} .
$$

The dimensionless product $W \alpha_{1} \equiv \Lambda$ is the inverse of the MacKinnon-Kramer parameter. ${ }^{30}$ We obtain $\alpha_{1}$ by increasing $L$ at constant $W$ until convergence is reached (typically for $L / W \simeq 10^{3}$ ). The large- $L$ limit is self-averaging but some improvement in statistical accuracy is reached by averaging

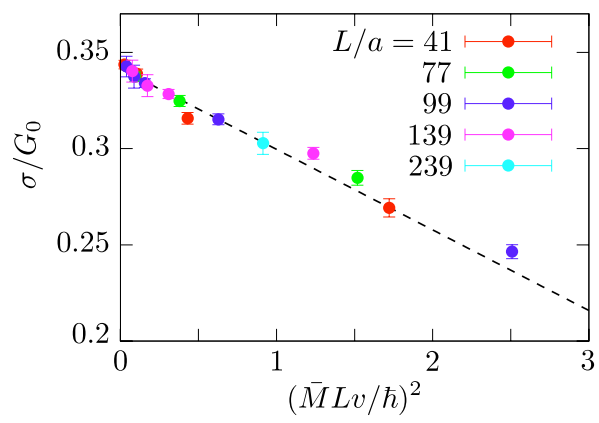

FIG. 3. (Color online) Plot of the average conductivity $\sigma$ versus $(\bar{M} L)^{2}$, for fixed $\delta M=2.5 \hbar / v a$ and $W / L=3$. The dashed line is a least-square fit through the data, consistent with critical exponent $\nu=1$. 


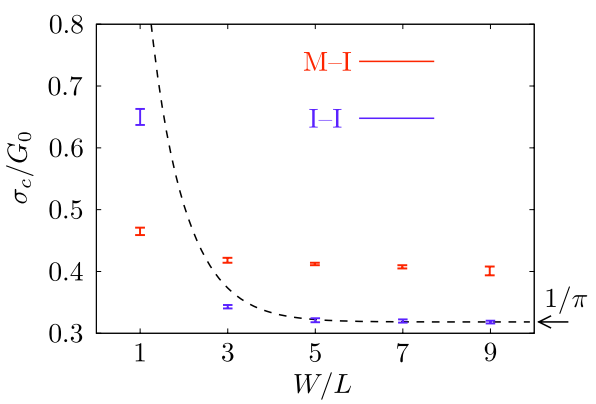

FIG. 4. (Color online) Dependence on the aspect ratio $W / L$ of the critical conductivity at the I-I transition $(\bar{M}=0, \delta M=2.5 \hbar / v a)$ and at the M-I transition $(\bar{M}=0.032 \hbar / v a, \delta M$ tuned to the transition). The dashed curve is the aspect-ratio dependence of the conductivity of ballistic massless Dirac fermions [Eq. (1.2)]. It describes the I-I transition quite well but not the M-I transition.

over a small number (10-20) of disorder realizations.

We seek the coefficients in the scaling expansion

$$
\Lambda=\Lambda_{c}+c_{1} W^{1 / \nu}\left(\bar{M}-M_{c}\right)+\mathcal{O}\left(\bar{M}-M_{c}\right)^{2},
$$

for fixed $\delta M$. The fit in Fig. 5 gives $\Lambda_{c}=0.03, \nu=1.05$, and $M_{c}=7 \cdot 10^{-4}$ consistent with the expected values ${ }^{10} \Lambda_{c}=0, \nu$ $=1$, and $M_{c}=0$.

\section{SCALING NEAR THE METAL-INSULATOR TRANSITION}

\section{A. Scaling of the conductivity}

To investigate the scaling near the metal-insulator transition, we increase $\delta M$ at constant $\bar{M}$. Results for the conductivity are shown in Fig. 6. In the metallic regime $\delta M$ $>\delta M_{c}$ the conductivity increases logarithmically with system size $L$, in accord with the theoretical prediction ${ }^{1,3}$

$$
\sigma / G_{0}=\frac{1}{\pi} \ln L+\text { const. }
$$

(See the dashed line in Fig. 6, upper panel.)

In the insulating regime $\delta M<\delta M_{c}$ the conductivity decays exponentially with system size while it is scale indepen-

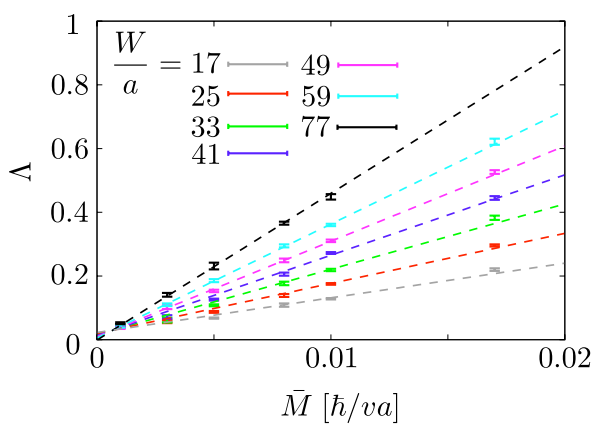

FIG. 5. (Color online) Plot of $\Lambda=W \alpha_{1}$ (with $\alpha_{1}$ the first Lyapunov exponent) as a function of $\bar{M}$ near the insulator-insulator transition, for fixed $\delta M=2.5 \hbar v / a$ and different values of $W$. The dashed lines are a fit to Eq. (4.2).

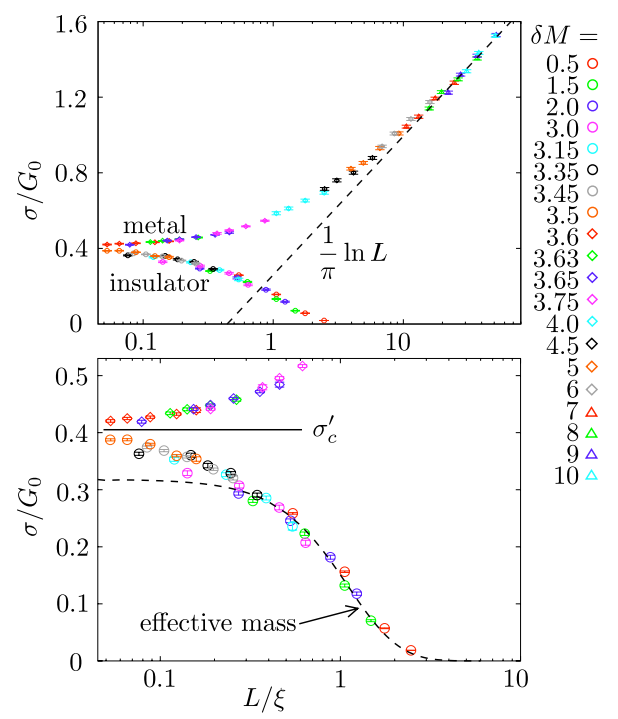

FIG. 6. (Color online) Average conductivity $\sigma$ at fixed average mass $\bar{M}=0.032 \hbar / v a$, as a function of system size $L$. (The two panels show the same data on a different scale.) The aspect ratio of the disordered strip is fixed at $W / L=5$. Data sets at different values of $\delta M$ (listed in the figure in units of $\hbar / v a$ ) collapse upon rescaling by $\xi$ onto a pair of curves in the metallic and insulating regimes. The metal-insulator transition has a scale invariant conductivity $\sigma_{c}^{\prime}$, larger than the value $G_{0} / \pi$ which follows from the effective-mass scaling (dashed curve in the lower panel). The upper panel shows that the conductivity in the metallic regime follows the logarithmic scaling [Eq. (5.1)].

dent at the critical point $\delta M=\delta M_{c}$. Data sets for different $\delta M$ collapse onto a single function of $L / \xi$ but this function is different from the effective-mass scaling $\sigma_{0}(L / \xi)$ of Eq. (1.1). (See the dashed curve in Fig. 6, lower panel.) This indicates that the effective-mass description, which applies well near the insulator-insulator transition, breaks down at large disorder strengths near the metal-insulator transition. The two transitions therefore have a different scaling behavior and can have different values of critical conductivity and critical exponent (which we denote by $\sigma_{c}^{\prime}$ and $\nu^{\prime}$ ).

Indeed, the critical conductivity $\sigma_{c}^{\prime}=0.41 G_{0}$ is significantly larger than the ballistic value $G_{0} / \pi=0.32 G_{0}$. Unlike at the insulator-insulator transition, we found no strong aspect-ratio dependence in the value of $\sigma_{c}^{\prime}$ (red data points in Fig. 4). To obtain the critical exponent $\nu^{\prime}$ we follow Ref. 31 and fit the conductivity near the critical point including terms of second order in $\delta M-\delta M_{c}$,

$$
\begin{aligned}
\sigma= & \sigma_{c}^{\prime}+c_{1} L^{1 / \nu^{\prime}}\left[\delta M-\delta M_{c}+c_{2}\left(\delta M-\delta M_{c}\right)^{2}\right] \\
& +c_{3} L^{2 / \nu^{\prime}}\left(\delta M-\delta M_{c}\right)^{2} .
\end{aligned}
$$

Results are shown in Fig. 7, with $\nu^{\prime}=1.02 \pm 0.06$. The quality of the multiparameter fit is assured by a reduced chisquared value close to unity $\left(\chi^{2}=0.95\right)$. Within error bars, this value of the critical exponent is the same as the value $\nu=1$ for the insulator-insulator transition. 


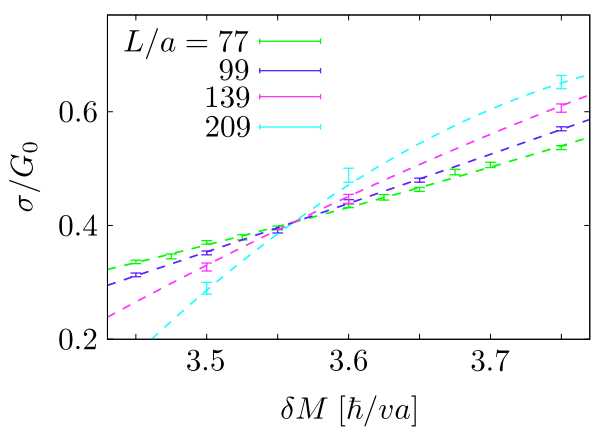

FIG. 7. (Color online) Plot of the average conductivity $\sigma$ as a function of $\delta M$ near the metal-insulator transition, for fixed $\bar{M}$ $=0.032 \hbar / v a$. The length $L$ is varied at fixed aspect ratio $W / L=3$. The dashed curves are a fit to Eq. (5.2).

\section{B. Scaling of the Lyapunov exponent}

As an independent measurement of $\nu^{\prime}$, we have investigated the finite-size scaling of the first Lyapunov exponent. Results are shown in Fig. 8. Within the framework of singleparameter scaling, the value of $\nu^{\prime}$ should be the same for $\sigma$ and $\Lambda$ but the other coefficients in the scaling law may differ,

$$
\begin{aligned}
\Lambda= & \Lambda_{c}+c_{1}^{\prime} L^{1 / \nu^{\prime}}\left[\delta M-\delta M_{c}^{\prime}+c_{2}^{\prime}\left(\delta M-\delta M_{c}^{\prime}\right)^{2}\right] \\
& +c_{3}^{\prime} L^{2 / \nu^{\prime}}\left(\delta M-\delta M_{c}^{\prime}\right)^{2} .
\end{aligned}
$$

Results are shown in Fig. 8, with $\nu^{\prime}=1.06 \pm 0.05$. The chisquared value for this fit is relatively large, $\chi^{2}=5.0$, but the value of $\nu^{\prime}$ is consistent with that obtained from the conductivity (Fig. 7).

\section{TRICRITICAL POINT}

As indicated in the phase diagram of Fig. 1, the tricritical point at $\bar{M}=0, \delta M=\delta M^{*}$ is the point at which the insulating phases at the two sides of the I-I transition meet the metallic phase. We have searched for this tricritical point by calculating the scale dependence of the conductivity $\sigma$ on the line $\bar{M}=0$ for different $\delta M$. Results are shown in Fig. 9 .

The calculated scale dependence is consistent with the identification of the point $\delta M^{*}=3.44 \hbar / v a$ as a repulsive

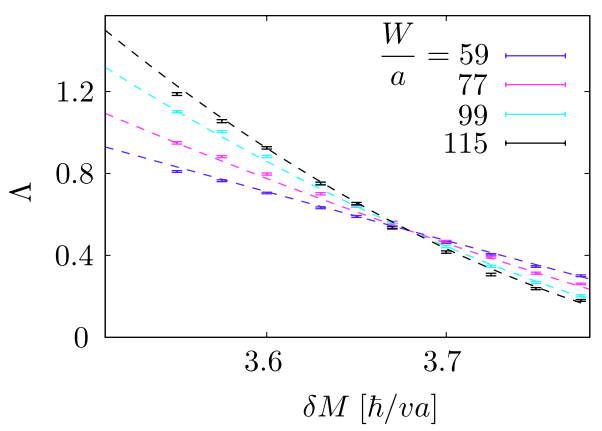

FIG. 8. (Color online) Plot of $\Lambda=W \alpha_{1}$ (with $\alpha_{1}$ the first Lyapunov exponent) as a function of $\delta M$ near the metal-insulator transition, for fixed $\bar{M}=0.032 \hbar v / a$ and different values of $W$. The dashed curves are a fit to Eq. (5.3).

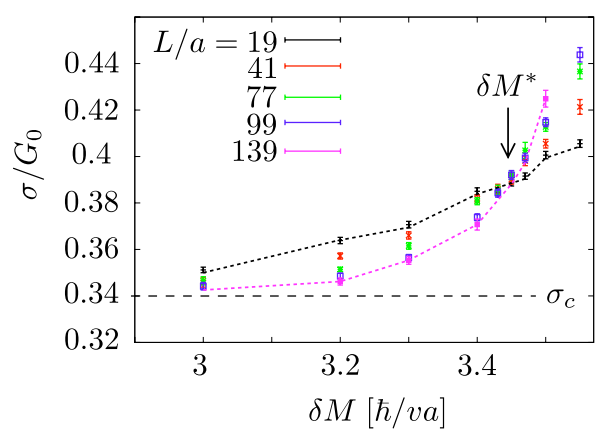

FIG. 9. (Color online) Conductivity $\sigma$ as a function of $\delta M$ on the critical line $\bar{M}=0$, for different values of $L$ at fixed aspect ratio $W / L=3$. (The dotted lines through data points are guides to the eyes.) The tricritical point $\delta M^{*}$ is indicated, as well as the scale invariant large- $L$ limit $\sigma_{c}$ for $\delta M<\delta M^{*}$.

fixed point. The conductivity increases with increasing $L$ for $\delta M>\delta M^{*}$ while for $\delta M<\delta M^{*}$ it decreases toward the scale invariant large- $L$ limit $\sigma_{c}$.

\section{DISCUSSION}

We have studied quasiparticle localization in symmetry class $B D$, by means of a lattice fermion model. ${ }^{17}$ The thermal quantum Hall effect ${ }^{2-4}$ in a chiral $p$-wave superconductor at weak disorder is in this universality class, as is the phase transition to a thermal metal ${ }^{3}$ at strong disorder.

For weak disorder our lattice model can also be used to describe the localization of Dirac fermions in graphene with

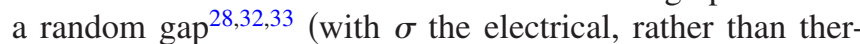
mal, conductivity and $G_{0}=4 e^{2} / h$ the electrical conductance quantum). The metallic phase at strong disorder requires Majorana bound states, ${ }^{2,5,6}$ which do not exist in graphene (symmetry class $D$ rather than $B D$ ). We therefore expect the scaling analysis in Sec. IV at the I-I transition to be applicable to chiral $p$-wave superconductors as well as to graphene while the scaling analysis of Sec. V at the M-I transition applies only in the context of superconductivity. (Here we disagree with Refs. 32 and 33, which maintain that the M-I transition exists in graphene as well.)

Our lattice fermion model is different from the network model $^{9}$ used in previous investigations ${ }^{10,11,15,16}$ but it falls in the same universality class so we expect the same critical conductivity and critical exponent. For the I-I transition analytical calculations ${ }^{1,14}$ give $\sigma_{c}=G_{0} / \pi$ and $\nu=1$, in agreement with our numerics. There are no analytical results for the M-I transition. We find a slightly larger critical conductivity $\left(\sigma_{c}^{\prime}\right.$ $\left.=0.4 G_{0}\right)$, which has the qualitatively more significant consequence that the effective-mass scaling which we have demonstrated at the I-I transition breaks down at the M-I transition (compare Figs. 2 and 6, lower panel).

We conclude from our numerics that the critical exponents $\nu$ at the I-I transition and $\nu^{\prime}$ at the M-I transition are both equal to unity within a $5 \%$ error margin, which is significantly smaller than the result $\nu=\nu^{\prime}=1.4 \pm 0.2$ of an earlier numerical investigation ${ }^{11}$ but close to the value found in later work by these authors. ${ }^{16}$ The logarithmic scaling [Eq. 
(5.1)] of the conductivity in the thermal metal phase, predicted analytically, ${ }^{1,3}$ is nicely reproduced by our numerics (Fig. 6, upper panel).

The nature of the tricritical point has been much debated in the literature. ${ }^{15,16}$ Our numerics indicates that this is a repulsive critical point (Fig. 9). This finding lends support to the simplest scaling flow along the I-I phase boundary, ${ }^{14}$ toward the free-fermion fixed point at $\bar{M}=0$ and $\delta M=0$.

In conclusion, we hope that this investigation brings us closer to a complete understanding of the phase diagram and scaling properties of the thermal quantum Hall effect. We now have two efficient numerical models in the $B D$ universality class, the Cho-Fisher network $\operatorname{model}^{9}$ studied previously and the lattice fermion model ${ }^{17}$ studied here. There is a consensus on the scaling at weak disorder, although some disagreement on the scaling at strong disorder remains to be resolved.

\section{ACKNOWLEDGMENTS}

We have benefited from discussions with A. R. Akhmerov, J. H. Bardarson, C. W. Groth, and M. Wimmer. This research was supported by the Dutch Science Foundation NWO/ FOM, by an ERC Advanced Investigator Grant, by the EU network NanoCTM, and by the ESF network EuroGraphene.

\section{APPENDIX: TRANSFER MATRIX FOR STAGGERED FERMIONS}

To make this paper self-contained, we give the staggeredfermion transfer matrix derived in Refs. 17, 24, and 25. The values $\Psi_{m, n}=\Psi\left(x_{m}, y_{n}\right)$ of the wave function at a lattice point are collected into a set of $N_{y}$-component vectors $\boldsymbol{\Psi}_{m}$ $=\left(\Psi_{m, 1}, \Psi_{m, 2}, \ldots, \Psi_{m, N_{y}}\right)$, one for each $m=1,2, \ldots, N_{x}$. The $N_{y} \times N_{y}$ transfer matrix $\mathcal{T}_{m}$ is defined by

$$
\boldsymbol{\Psi}_{m+1}=\mathcal{T}_{m} \boldsymbol{\Psi}_{m}
$$

The transfer matrix $\mathcal{T}$ through the entire strip is then the product of the $\mathcal{T}_{m}$ 's.

The differential operators in the Dirac Hamiltonian [Eq. (2.2)] are discretized by

$$
\begin{aligned}
& \partial_{x} \Psi \rightarrow \frac{1}{2 a}\left(\Psi_{m+1, n}+\Psi_{m+1, n+1}-\Psi_{m, n}-\Psi_{m, n+1}\right), \\
& \partial_{y} \Psi \rightarrow \frac{1}{2 a}\left(\Psi_{m, n+1}+\Psi_{m+1, n+1}-\Psi_{m, n}-\Psi_{m+1, n}\right),
\end{aligned}
$$

and the mass term is replaced by

$$
M \sigma_{z} \Psi \rightarrow \frac{1}{4} M_{m, n} \sigma_{z}\left(\Psi_{m+1, n}+\Psi_{m+1, n+1}+\Psi_{m, n}+\Psi_{m, n+1}\right)
$$

with $M_{m, n}=M\left(x_{m}+a / 2, y_{n}+a / 2\right)$. The zero-energy Dirac equation $H_{\text {Dirac }} \Psi=0$ is applied at the points $\left(x_{m}+a / 2, y_{n}\right)$ by averaging the terms at the two adjacent points $\left(x_{m}\right.$ $+a / 2, y_{n} \pm a / 2$ ). (This is the staggered lattice construction introduced by Kogut and Susskind to avoid the fermion doubling problem. ${ }^{34}$ )

The resulting finite difference equation can be written in a compact form with the help of the $N_{y} \times N_{y}$ tridiagonal matrices $\mathcal{J}, \mathcal{K}$, and $\mathcal{M}^{(m)}$, defined by the following nonzero elements:

$$
\begin{gathered}
\mathcal{J}_{n, n}=1, \quad \mathcal{J}_{n, n+1}=\mathcal{J}_{n, n-1}=\frac{1}{2}, \\
\mathcal{K}_{n, n+1}=\frac{1}{2}, \quad \mathcal{K}_{n, n-1}=-\frac{1}{2}, \\
\mathcal{M}_{n, n}^{(m)}=\frac{1}{2}\left(M_{m, n}+M_{m, n-1}\right), \quad \mathcal{M}_{n, n+1}^{(m)}=\frac{1}{2} M_{m, n}, \\
\mathcal{M}_{n, n-1}^{(m)}=\frac{1}{2} M_{m, n-1} .
\end{gathered}
$$

In accordance with the periodic boundary conditions in the transverse direction, the indices $n \pm 1$ should be evaluated modulo $N_{y}$.

The discretized Dirac equation is expressed in terms of the matrices [Eqs. (A5)-(A7)] by

$$
\begin{aligned}
\frac{1}{2 a} \mathcal{J}\left(\boldsymbol{\Psi}_{m+1}-\boldsymbol{\Psi}_{m}\right)= & \left(-\frac{i}{2 a} \sigma_{z} \mathcal{K}-\frac{1}{4} v^{2} \sigma_{y} \mathcal{M}^{(m)}\right) \\
& \times\left(\boldsymbol{\Psi}_{m}+\boldsymbol{\Psi}_{m+1}\right) .
\end{aligned}
$$

Rearranging Eq. (A8) we arrive at Eq. (A1) with the transfer matrix

$$
\begin{aligned}
\mathcal{T}_{m}= & \left(\mathcal{J}+i \sigma_{z} \mathcal{K}+\frac{1}{2} v^{2} a \sigma_{y} l M^{(m)}\right)^{-1} \\
& \times\left(\mathcal{J}-i \sigma_{z} \mathcal{K}-\frac{1}{2} v^{2} a \sigma_{y} \mathcal{M}^{(m)}\right) .
\end{aligned}
$$

Particle-hole symmetry for the zero-energy-transfer matrix requires

$$
\sigma_{x} \mathcal{T}_{m}^{*} \sigma_{x}=\mathcal{T}_{m},
$$

which is satisfied by Eq. (A9). Current conservation requires

$$
\mathcal{T}_{m}^{*} J_{x} \mathcal{T}_{m}=J_{x}
$$

which holds for the discretized current operator

$$
J_{x}=\frac{1}{2} v \sigma_{x} \mathcal{J} .
$$

For a uniform mass $M_{m n}=M$, we may calculate the eigenvalues $e^{i k_{x} a}$ of $\mathcal{T}_{m}$ analytically. This gives the dispersion relation

$$
\tan ^{2}\left(k_{x} a / 2\right)+\tan ^{2}\left(k_{y} a / 2\right)+(M a v / 2 \hbar)^{2}=0
$$

with $k_{y}=2 \pi l / N_{y}, l=1,2, \ldots, N_{y}$, in accord with Eq. (3.1) at zero energy. 
${ }^{1}$ F. Evers and A. D. Mirlin, Rev. Mod. Phys. 80, 1355 (2008).

${ }^{2}$ N. Read and D. Green, Phys. Rev. B 61, 10267 (2000).

${ }^{3}$ T. Senthil and M. P. A. Fisher, Phys. Rev. B 61, 9690 (2000).

${ }^{4}$ A. Vishwanath, Phys. Rev. Lett. 87, 217004 (2001).

${ }^{5}$ M. Bocquet, D. Serban, and M. R. Zirnbauer, Nucl. Phys. B 578, 628 (2000).

${ }^{6}$ N. Read and A. W. W. Ludwig, Phys. Rev. B 63, 024404 (2000).

${ }^{7}$ G. E. Volovik, JETP Lett. 70, 609 (1999).

${ }^{8}$ V. Gurarie and L. Radzihovsky, Phys. Rev. B 75, 212509 (2007).

${ }^{9}$ S. Cho and M. P. A. Fisher, Phys. Rev. B 55, 1025 (1997).

${ }^{10}$ J. T. Chalker, N. Read, V. Kagalovsky, B. Horovitz, Y. Avishai, and A. W. W. Ludwig, Phys. Rev. B 65, 012506 (2001).

${ }^{11}$ V. Kagalovsky and D. Nemirovsky, Phys. Rev. Lett. 101, 127001 (2008).

${ }^{12}$ M. I. Katsnelson, Eur. Phys. J. B 51, 157 (2006).

${ }^{13}$ J. Tworzydło, B. Trauzettel, M. Titov, A. Rycerz, and C. W. J. Beenakker, Phys. Rev. Lett. 96, 246802 (2006).

${ }^{14}$ A. W. W. Ludwig, M. P. A. Fisher, R. Shankar, and G. Grinstein, Phys. Rev. B 50, 7526 (1994).

${ }^{15}$ A. Mildenberger, F. Evers, A. D. Mirlin, and J. T. Chalker, Phys. Rev. B 75, 245321 (2007).

${ }^{16}$ V. Kagalovsky and D. Nemirovsky, Phys. Rev. B 81, 033406 (2010).

${ }^{17}$ J. Tworzydło, C. W. Groth, and C. W. J. Beenakker, Phys. Rev. B 78, 235438 (2008). This paper considers scattering of staggered fermions by a potential $V$ rather than by a mass $M$ but one simply needs to replace $V$ by $v^{2} M \sigma_{z}$ to obtain the transfer matrix required here.

${ }^{18}$ C. Kallin and A. J. Berlinsky, J. Phys.: Condens. Matter 21,
164210 (2009).

${ }^{19}$ S. Tewari, S. Das Sarma, C. Nayak, C. Zhang, and P. Zoller, Phys. Rev. Lett. 98, 010506 (2007).

${ }^{20}$ M. Sato, Y. Takahashi, and S. Fujimoto, Phys. Rev. Lett. 103, 020401 (2009).

${ }^{21}$ J. Sau, R. Lutchyn, S. Tewari, and S. Das Sarma, Phys. Rev. Lett. 104, 040502 (2010).

${ }^{22}$ P. Lee, arXiv:0907.2681 (unpublished).

${ }^{23}$ J. Alicea, Phys. Rev. B 81, 125318 (2010).

${ }^{24}$ R. Stacey, Phys. Rev. D 26, 468 (1982).

${ }^{25}$ C. M. Bender, K. A. Milton, and D. H. Sharp, Phys. Rev. Lett. 51, 1815 (1983).

${ }^{26}$ B. Kramer, T. Ohtsuki, and S. Kettemann, Phys. Rep. 417, 211 (2005).

${ }^{27}$ M. Wimmer, A. Akhmerov, M. Medvedyeva, J. Tworzydło, and C. Beenakker, arXiv:1002.3570 (unpublished).

${ }^{28}$ J. H. Bardarson, M. V. Medvedyeva, J. Tworzydło, A. R. Akhmerov, and C. W. J. Beenakker, Phys. Rev. B 81, 121414(R) (2010).

${ }^{29}$ J. H. Bardarson, J. Tworzydło, P. W. Brouwer, and C. W. J. Beenakker, Phys. Rev. Lett. 99, 106801 (2007).

${ }^{30}$ A. MacKinnon and B. Kramer, Phys. Rev. Lett. 47, 1546 (1981).

${ }^{31}$ Y. Asada, K. Slevin, and T. Ohtsuki, Phys. Rev. B 70, 035115 (2004).

${ }^{32}$ K. Ziegler, Phys. Rev. Lett. 102, 126802 (2009); Phys. Rev. B 79, 195424 (2009).

${ }^{33}$ K. Ziegler and A. Sinner, Phys. Rev. B 81, 241404(R) (2010).

${ }^{34}$ J. Kogut and L. Susskind, Phys. Rev. D 11, 395 (1975). 